\title{
The study of the movement of a piece on the camera crushing in network type bases as applied to the workflow of the FCC
}

\author{
Yuliya Lagunova ${ }^{1}$, Victor Shestakov $^{1}$, and Nazira Ibrayeva $^{1,2}$ \\ ${ }^{1}$ Ural State Mining University, 620144 Ekaterinburg, Russia \\ ${ }^{2}$ Karaganda State Technical University, 100027 Karaganda, Republic of Kazakhstane
}

\begin{abstract}
The paper considers a software-modelling structure for solving the problem of crushing of solid materials, mountain mineral raw materials of different strength and composition. The task is to study the movement of a piece along the crushing chamber of a cone crusher, the stages of destruction of pieces are considered. Setting the main parameters of the FCC, the working process of the crusher and the parameters of the destruction of pieces in the crushing chamber. The study of the basic characteristics of the movement process, the interaction and destruction of pieces is the main aspect of the work process. More precisely, the issues were examined, on the basis of which the dependencies were compiled, which are used in the algorithm of the model of the working process of FCC at the beginning of the cycle of the mode of movement. One of the processes considered in the article is squeezing a piece between the armors with a subsequent decrease in the distance between the armors.
\end{abstract}

\section{Introduction}

Ore preparation includes the process of crushing the ore, the main task of which is further enrichment. Crushing is the process of material destruction that is carried out in crushers.

Research in the field of rock crushing has been conducted for a long time. Studies have shown that the destruction of rocks by different impacts is accompanied by different reactions, so it is necessary to distinguish between the crushing of rocks during destruction by compression, compression, impact, abrasion, etc[1,2].

The method of destruction is determined by the force acting on the crushed piece. In addition, the crushing process is affected by the number of simultaneously crushed pieces. There is a distinction between splitting a single piece "piece by piece" and splitting "in a layer" (piece by piece). Numerous studies of the working process have proved that in FCC crushers, the material is destroyed by compression, and single pieces are crushed.

Objectives of the study[3-14]:

- the workflow definition FCC;

- determining the values of geometric parameters;

- study of the movement of pieces in the crushing chamber. 
Purpose to develop a method for calculating the workflow of cone crushers of fine crushing for use in creating an intelligent crusher based on the construction of neural networks.

\section{Research method}

The use of computer systems in industry and mining is an integral part of the entire production system. The use of artificial intelligence in the study of the working process of crushing, taking into account the movement of the piece in the crushing chamber, is an urgent task.

Using the Excel program, the process of operation of a cone crusher is considered, and modeling of technological and software components is performed. A universal criterion for creating a database table "data for calculation" (Fig. $1 \mathrm{a}, \mathrm{b}$ ) is an element that reflects the main content of the table, which is reflected in its name, so the main field is called "data for calculating a cone crusher" and it will contain its description. Next, to calculate the trajectory of the piece, you need to create: "table with FCC data", "calculation of basic parameters", "data verification", "calculation results".

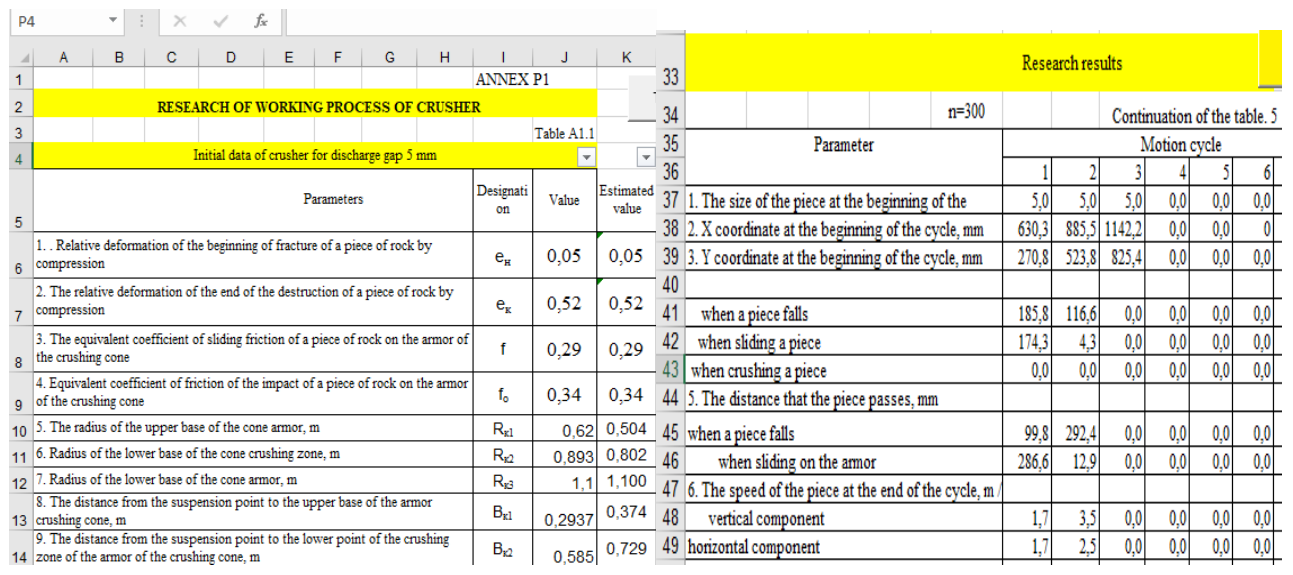

Fig. 1. a) table with data for calculating FCC, b) table with data for FCC, C) checking the source data.

Now the filled-in table cells automatically switch to checking the source data (Fig. 1, b). To do this, open the table "checking source data", using the "Open" mode and then "Checking".

The most important aspect is the tables that need to be linked so that they represent logically related unified data that can be processed. By connecting all the tables, we can assume that our information base for the solution has been created. In turn, such a program can transform into a model for trajectory analysis of the solution of a given problem and the task set for the program, created using logically connected tables. In this case, the created tables and entered data set the task to solve, determine and investigate the trajectory of the piece movement along the crushing chamber.

When a piece of crushing moves, several crushing cycles occur, but these cycles depend on the size of the piece. The property of crushing begins to appear after the relative deformation of a certain horn is exceeded. The beginning of manifestation is associated with the destruction of the piece. The piece ceases to exist as such, turns into its own fragments.

When pressing, there is no destruction, and the elements of the crusher are subjected to increased loads, which leads to increased wear, so during the crushing process, it is 
necessary to exclude cases of pressing pieces. This provision was used for the calculation of dependencies. In addition to excluding pressing, for the normal crushing process, it is necessary to ensure the free entry of crushed pieces into the crushing chamber.

The movement of parts consists of stages[14]:

- free fall of a piece after it enters the chamber before meeting the cone armor;

- relative movement (sliding) of the piece on the armor of the cone and its axial movement together with the cone to the bowl until the end of the phase of convergence of the cone with the bowl;

- moving the piece together with the cone armor to the bowl;

- stopping the piece due to its coming into contact with both armor and crushing the piece;

- unloading of fragments from the crushing chamber starting with the next phase of removing the cone from the bowl.

Pieces that are close to the size of the discharge slot in a closed position will immediately start moving when the receiving slot is opened, perform a free fall to meet the cone armor and then slide along it. Their size is smaller than the size of the crushing chamber in the closed state, so they will not stop and during the second cycle they will start falling at the speed that was reached in the first cycle. If the length of the crushing chamber is not sufficient, such pieces do not have time to be crushed in a parallel zone, and the crusher does not have time to be crushed.

In crushers with insufficient length of the crushing chamber, a certain proportion of the output product is larger than the size of the discharge slot.

To avoid the possibility of large pieces leaving without crushing, it is necessary to ensure that the size of the crushing chamber is such that even small pieces pass through the crushing chamber in two complete cycles of cone movement. All research and solutions are listed in tables in Excel, which in the future will allow us to solve the problem we have set.

Figure 4 shows a trajectory with two crushing cycles. All large pieces of the discharge slot in the closed position will be caught in the calibration zone and crushed.
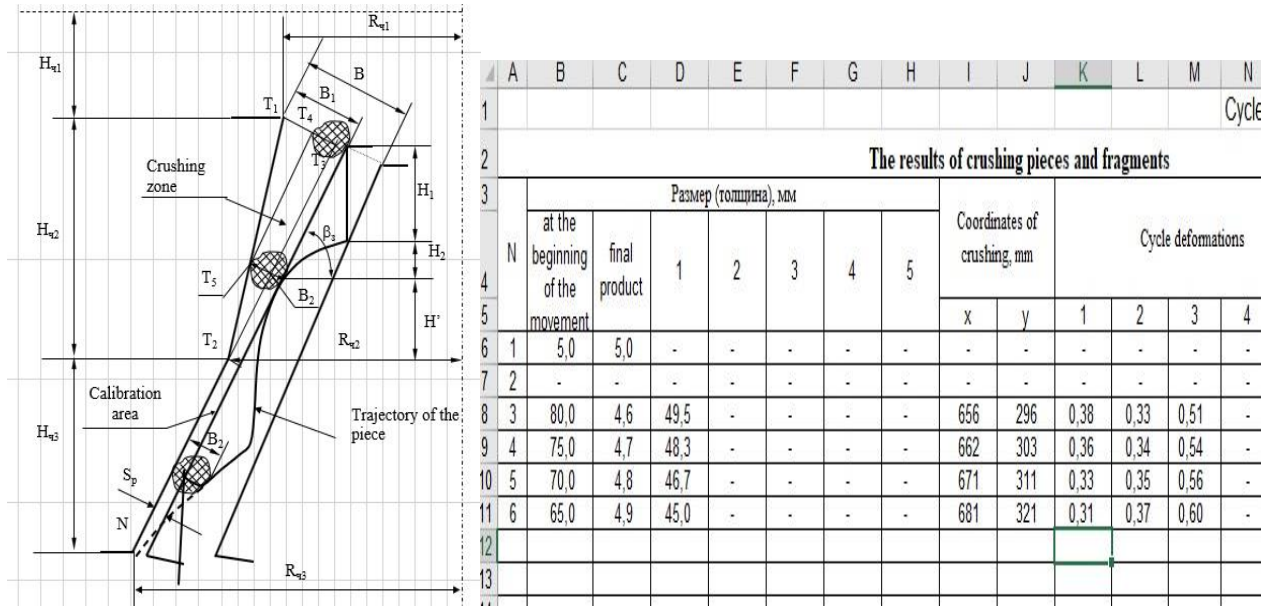

Fig. 4. a) Diagram for calculating the parameters of the crushing chamber b) Summary table for the result crushing parts.

The initial data for building the model are: the dimensions of the cone and bowl, the angle of nutation, the frequency of swinging of the cone, the initial position of the cone, the size of the initial piece and its characteristic (equivalent coefficient of friction), the position of the piece at the beginning of movement, the integration step. 
Dimensions of the cone and bowl armor $\omega, \psi \mathrm{H}, \mathrm{vH}$, хк, ук,dк. The model is based on the equations of motion of a piece and a crushing cone. The equations are solved using numerical methods discretely through the integration step $\Delta \mathrm{t}$.

The algorithm is constructed as follows.

At the beginning of the calculation cycle, the "Drop" movement mode is assumed, the rotation angle is assumed to be equal to the initial one, that is, the variable that starts the movement cycle of the piece is assigned $1 . \psi=\psi \mathrm{H}, \mathrm{i}=1$

Expressions that implement the movement of the cone are solved, and the deviation of the cone from the closed state Qt is determined through the integration step, the coordinates of the points of the cone armor, the angle of inclination of the sections of the cone armor, the coordinates of the vertical projection of the piece on the UBR cone armor, the distance between the cone and bowl armor at the location of the dt piece "The position of the cone».

Checks whether the piece enters the receiving slot of the crusher at its position according to the condition $\mathrm{d} \kappa<\Delta \mathrm{T}$. If the condition is not met, that is, the piece does not enter the receiving slot, then the "Delay" mode is set, the piece stops at the edge of the cone armor - its speed is assumed to be zero.

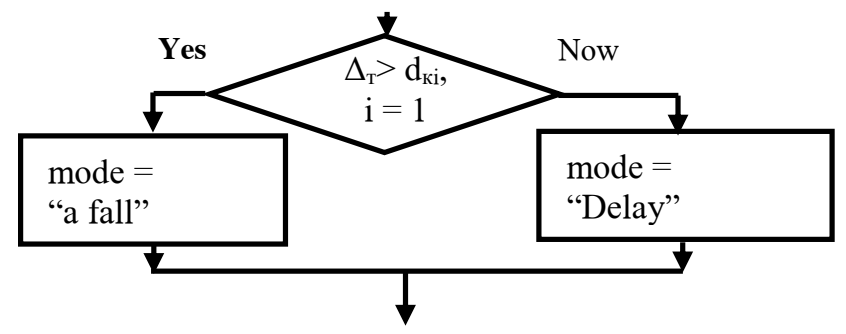

If a piece enters the receiving slot, then the equations of motion of the piece when it falls are solved and the coordinates of the piece $\mathrm{Xk}, \mathrm{Yk}$ and its speed $\mathrm{V}_{\mathrm{C}}$ are determined at the integration step VB "Drop of a piece». A fall $x_{\mathrm{K}}, y_{\mathrm{K}}, v_{\mathrm{K}}$.

When solving the equations of motion of a piece during a fall, it is simultaneously checked whether the piece of armor has reached the cone under the condition Yк $\leq \mathrm{V}$. If the condition is met (the piece has not reached the armor), the mode remains the same - «a fall» and when the condition is stopped, the mode changes to "Sliding".
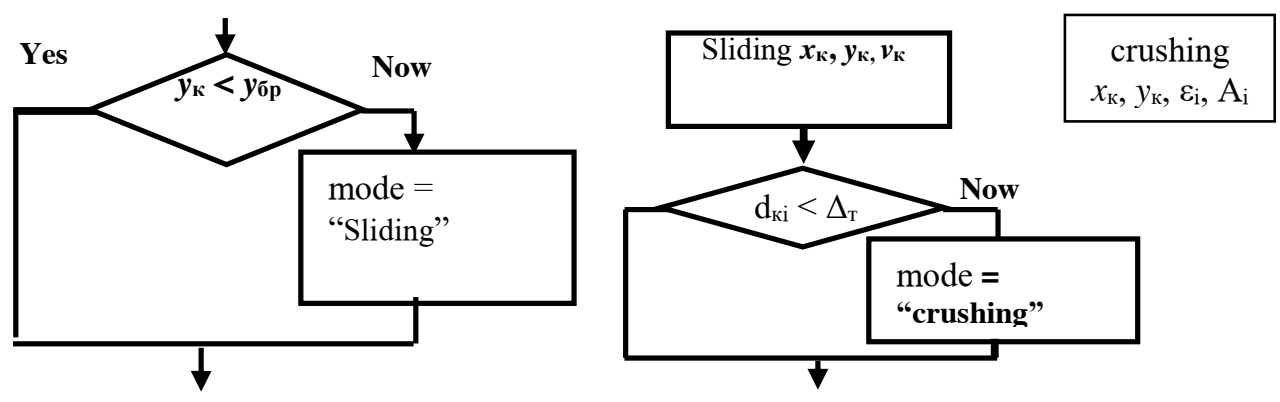

When changing the mode from falling to sliding, the initial value of the speed of movement of the piece along the cone armor is calculated and expressions implementing the sliding process begin to be resolved. During the decision process, the current position of the piece on the cone's armor is determined (coordinates of the piece Хк, Үк) and the condition for its further movement along the cone's armor $\mathrm{d \kappa}<\Delta \mathrm{T}$ is checked (whether the distance between the armor has decreased to the size of the piece). While the condition is 
met, the mode remains "Sliding", and if the condition is violated, the mode switches to" Crushing", "Moving the piece together with the armor of the cone".

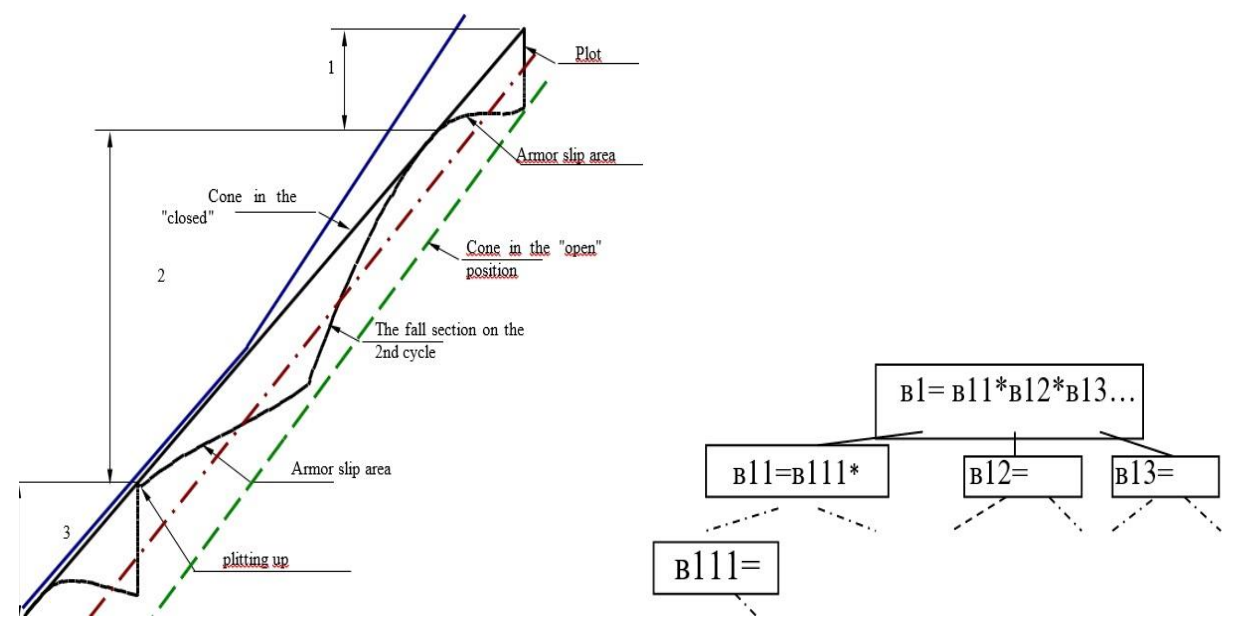

Fig. 5. Algorithm of the FCC workflow model.

The maximum size of a piece after crushing is equal to the distance between the armor at the point of crushing. The expression provides a change in the angle of the gate of the eccentric by a step $\omega \Delta \mathrm{t}$, and the condition checks the completion of the movement cycle.

$$
\text { dкі }=\Delta \mathrm{T} \quad \boldsymbol{\psi}=\boldsymbol{\psi}+\boldsymbol{\omega} \Delta \mathrm{t} \quad \boldsymbol{\psi}<\mathbf{2 \pi}
$$

At the end of the first rotation of the eccentric until the receiving zone is completely closed, the solution is repeated to track the further movement of fragments of crushing pieces. If the condition

$\mathrm{d} \kappa<\Delta \mathrm{T}$ was fulfilled during the entire period of the cone swing, i.e. there was no crushing, then the transition to the next cycle occurs with the speed reached during the previous cycle. Cycles are repeated until the exit from the crushing chamber of fragments according to the condition, the values of relative deformations, energy consumption, and the thickness of the output product are output to the output forms.

In the sequence cycle of the trajectory of the piece through the crushing chamber due to accurate data and research workflow the basic model allowed to reveal the exact result. The algorithm for modulating the FCC workflow at the beginning of the calculation cycle, the movement mode is adopted, which allowed us to study the working process of the crushing chamber. The results are listed in the "Summary table of the results of crushing parts". An analysis created in a combination program where all the elements of a piece's movement are shown in stages. The use of the program easily varies from the modulating to the control, where the tables in turn are supplemented with lines for storing and processing data of the FCC. Thus, the system is monitored from the beginning of the movement, which will prevent sharp increases in loads on the mechanism elements.

\section{Results}

The following results were obtained:

- the analysis of the movement of the piece through the crushing chamber as an object of research. It is shown that when studying the work of the FCC, it is necessary to use a mathematical modeling program with direct use of neural networks, for further analysis and solving the problems of moving a piece; 
- developed a scheme for the movement of a piece through the crushing chamber in Excel and an algorithm for solving the problem using a computer for the working process of FCC;

- based on the obtained data and the developed model, a neural network connection was created between the program and the equipment simulated by a mathematical model and a decision algorithm that is able to track the movement of a piece through the crushing chamber with specified parameters, checking the data and calculation results.

\section{Findings}

The article deals with the actual problem of creating a simulation process of the FCC operation for the movement of a piece through the crushing chamber. To study the movement of a piece through the crushing chamber, the Excel program was used as a basis.

In addition, research work was carried out aimed at the optimal solution of problems that arise during the operation of the equipment, namely, when the piece moves through the crushing chamber.

\section{References}

1. I.I. Blechman, N.A. Ivanov, Ore processing, 2, 35 (1977)

2. I.I. Blekhman, N.A.Ivanov, Ore enrichment, 1, 24 (1979)

3. B.D. Kotelnikov, Sb. trudov SGI, 37 (1983)

4. Yu.a. Lagunova, Izv.vuzov. Construction, 1, 116 (1998)

5. A.J. Lynch, TRANS, 343 (1981)

6. V.A. Maslennikov, Izv.vuzov. Mountain journal, 12, 74 (1987)

7. V.A. Maslennikov, Izv. The Ural mining Institute, 4, 9 (1993)

8. Yu.a. Muizemnek, Izv.vuzov. Mining journal, 7, 68 (1978)

9. S.A. Chervyakov, Mining machines and automation, 8, 27 (2004)

10. Yu.a. Lagunova, V.S. Bochkov, the energy component of the properties of the material Shredding layer abstract of lectures on mechanical engineering, 577 (2020)

11. California Tang, $\mathrm{HN} \mathrm{Xu}$, sq. kou, P. Lindquist, N. Yu. International journal of rock mechanics and mining Sciences, 38 (8), 1147 (2001)

12. J. Felix, M. Mazur, SGEM, 17 (11), 1081

13. A.P. Komissarov, V.S. Shestakov, Yu.A. Lagunova, N.S. Malybaev, N.R. Ibrayeva, Computer program. Study of the parameters of the working process and the movement of pieces along the crushing chamber of the cone crusher, (2020) 